\title{
Test de Percepción de Diferencias: Estudio normativo del desempeño atencional EN ESCOLARES ARGENTINOS*
}

\section{Perception of differences test (faces): Normative study of attentional PERFORMANCE IN ARGENTINE STUDENTS}

\author{
Juan Manuel Monteoliva**, Mariana Carrada ${ }^{* * *}$ y Mirta Susana Ison ${ }^{* * * *}$
}

\begin{abstract}
*Trabajo realizado en el marco de los proyectos PIP- 11220100100347 Desarrollo de funciones socio-cognitivas en escolares en situación de vulnerabilidad (CONICET-INCIHUSA) otorgado a la Dra. Mirta S. Ison.

**Doctor en Medio Ambiente Visual e lluminación Eficiente. Becario Post Doctoral del Consejo Nacional de Investigaciones Científicas y Técnicas (CONICET). E-Mail: jmonteoliva@mendoza-conicet.gob.ar

${ }^{* * *}$ Doctora en Psicología. Investigadora del Instituto de Investigaciones de la Facultad de Psicología de la Universidad del Aconcagua (UDA) y Profesora de la Facultad de Ciencias de la Salud de la Universidad de Mendoza.

E-Mail: mcarrada@gmail.com

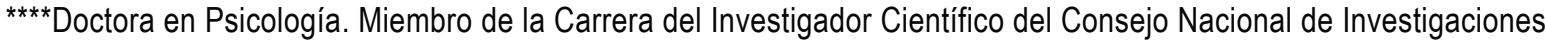
Científicas y Técnicas (CONICET) y Profesora de la Cátedra de Psicología del Desarrollo en la Facultad de

Psicología de la Universidad del Aconcagua (UDA). E-Mail: mison@mendoza-conicet.gob.ar

Los autores agradecen el apoyo profesional y humano recibido al Grupo de Psicología Evolutiva y Educacional del Instituto de Ciencias Humanas Sociales y Ambientales (INCIHUSA) y al Grupo de lluminación Natural del Instituto de Ambiente, Hábitat y Energía (INAHE), ambos del Centro Científico Tecnológico Mendoza. Instituto de Ciencias Humanas Sociales y Ambientales (INCIHUSA - CONICET).

Av. Ruiz Leal S/N. Parque General San Martín. (CP 5500) Mendoza, República Argentina.
\end{abstract}

\section{RESUMEN}

La atención juega un rol clave en el desempeño escolar de los niños. Numerosas investigaciones han informado que la capacidad atencional se correlaciona positivamente con las competencias de lectura, escritura y habilidades matemáticas en escolares primarios. En este marco surge la necesidad de evaluar las habilidades atencionales de los niños en el ámbito educativo con instrumentos válidos, confiables y adaptados a la población escolar. El propósito del trabajo realizado fue elaborar los datos normativos del Test de Percepción de Semejanzas y Diferencias (CARAS) y sus indicadores de desempeño atencional. Esta prueba es utilizada para evaluar la atención focalizada y sostenida y la aptitud para percibir, rápida y correctamente, semejanzas, diferencias y patrones estimulantes parcialmente ordenados. La investigación fue descriptiva y se empleó un diseño no experimental, seccionaltransversal. El análisis se hizo con una muestra estandarizada por muestreo aleatorio estratificado de 4.462 alumnos, de establecimientos escolares públicos de nivel primario del Gran Mendoza (República Argentina) de 7 a 12 años de edad. Como resultado se obtuvieron puntuaciones correspondientes al desempeño atencional: eficacia atencional (EA), eficiencia atencional (FA) y rendimiento atencional (RA), en tiempos parciales de evaluación ( $\mathrm{t} 1$ y t2) y tiempo total (T), según sexo y edad. Luego se determinaron las frecuencias acumuladas que posteriormente fueron transformadas a rangos percentilares. Con este tipo de estudio normativo se pretende brindar en los diferentes indicadores de desempeño atencional, una mayor precisión diagnóstica en la evaluación del mecanismo atencional en niños. 
Palabras clave: Desempeño atencional; Test de Percepción de Diferencias (CARAS); Eficacia atencional; Eficiencia atencional; Rendimiento atencional; Niños.

\section{ABSTRACT}

Attention plays a key role in school performance of children. Different studies have reported that the attentional capacity is positively correlated with the skills of reading, writing and math in primary school. In the teaching-learning process, a child uses different forms of attention. In one task, the child selects relevant information and inhibits the others. Perhaps, should divide his attention and switch his focus of attention between two or more sources of information, holding -intentionallythese activities for a specified time. In this context, it is important evaluate the attentional abilities of children in education with valid, reliable and adapted instruments to the school population. The main perceptual-attentional skills used in assessing attentional tasks are: perception of differences, visual discrimination, visual integration and perceptual speed. These tasks can be characterized as simple, yet include a lot of tests, which requires a relatively large time to be realized. In this way become continuous performance tasks in which the subject must locate significant stimulus to a wider set of stimuli acting distracting way. The purpose of this work was the development of normative data of performance indicators in Perception Test Similarities and Differences or FACES. This test is used to evaluate the focused and sustained attention and the ability to perceive, quickly and correctly, similarities, differences and partially ordered stimulating patterns. As a main feature, the CARAS, has 60 blocks stimuli each composed of three schematic drawings of faces (with elementary strokes representing the mouth, eyes, eyebrows and hair), one of which is different. The task is to determine the different side and cross it off. The research was descriptive and cross-sectional non-experimental design was used. The anal- ysis was performed on a standardized sample stratified random sample of 4.462 students between 7 and 12 years of public primary schools of Mendoza (República Argentina). As a result are obtained performance attentional scores: attentional efficacy (AE), attentional efficiency (AE) and attentional yield (AY) in partial evaluation time ( $\mathrm{t} 1$ and $\mathrm{t} 2$ ) and total time (T), for each sex and age. These attentional assessment indicators are proposed by the authors in previous work. Mathematical expressions derived from these indicators, emerged from the chronological review of the progress made in this assessment tool and the need to know more accurately the capabilities of the subjects tested. In this context, the term attentional performance is introduced as an inclusive concept of attentional efficiency, attentional efficiency and attentional yield of a subject in visual search tasks. Attentional efficiency allows to evaluate the accuracy with which a child discriminate stimuli within a series of similar stimuli; attentional efficiency (AF) is defined as the cognitive ability to regulate and optimize the attentional mechanism to select and maintain attention for an extended period of time, using minimal resources of time available. Moreover, attentional yield (AY) is the product resulting from the level of effectiveness and efficiency obtained in selection and sustained attentional task. Then, the cumulative frequencies, which were subsequently transformed to percentile ranks, were determined. With this kind of normative study is to provide, in different attentional performance indicators, greater diagnostic accuracy in assessing attentional mechanism in children. The attentional capacity is key to proper cognitive functioning, thus its evaluation is relevant for the purposes of precise diagnosis and adequate psycho-educational intervention programs that interrupt the cycle of failure and frustration helping to increase the feeling of self-efficacy in the school.

Key words: Attentional performance; Test on Perception of Differences (FACES); Attentional efficacy; Attentional efficiency; Attentional yield; Children.

\section{INTRODUCCIÓN}

La complejidad conceptual, neuroanatómica y neurofuncional de la atención hace que no pueda ser reducida a una simple defi- 
nición ni ser evaluada con un solo test. Es decir, la atención entendida como un mecanismo de control, presenta un rol fundamental en la organización jerárquica de los procesos encargados de elaborar la información, tales como la percepción, la memoria de trabajo, la flexibilidad cognitiva y en los procesos motivacionales y emocionales (Álvarez \& Trápaga, 2005; Ison, Greco, Korzeniowski \& Morelato, 2015; Petersen \& Posner, 2012; Rosselló i Mir, 1998). Se conceptualiza como un mecanismo neurocognitivo que controla el procesamiento de la información a través de la activación o inhibición de los procesos psicológicos para alcanzar eficazmente la ejecución de una tarea determinada y como tal, está compuesto por redes neuronales cuyas estructuras se activan de acuerdo a la operación de selección, mantenimiento y / o distribución implicada en la actividad atencional. La necesidad de un control voluntario, intencional y consciente de los procesos cognitivos, originados a partir de lo percibido y de la regulación deliberada del comportamiento o de la acción, dependen del mecanismo atencional (Fuentes Melero, 2004; Fuentes Melero \& Campoy, 2008; Fuentes Melero \& García Sevilla, 2008). Las funciones más específicas de dicho mecanismo son las de ser más receptivos a los sucesos del ambiente, llevar a cabo un adecuado análisis de la realidad y facilitar la activación y el funcionamiento de otros procesos psicológicos para ejecutar eficazmente las tareas (Ison \& Carrada, 2011).

En el aprendizaje escolar, la atención es una función neuropsicológica de suma importancia ya que contribuye a la concentración del alumno y posibilita la adquisición de conocimientos (Álvarez, González-Castro, Núñez, González Pienda, Alvarez \& Bernardo, 2007a, 2007b; Colombo, 2001; Gómez Pérez \& Ostrosky Solís, 2006; Posner \& Rothbart, 2014). Una revisión de la literatura sobre el tema (Cassandra \& Reynolds, 2005; Porte1lano, 2007) indica que el desarrollo de la atención debe ser considerado como un proceso con diferentes funciones y madurando en diferentes momentos, lo que hace referencia a la existencia de diversos períodos sensibles, considerados como ventanas de tiempo en las cuales la plasticidad cerebral está incrementada, posibilitando que el funcionamiento cognitivo sea promovido por la experiencia (Armstrong et al., 2006, citado en Korzeniowski, 2011). Estos períodos coinciden con la etapa de escolarización, por lo que resulta interesante analizar la relación entre el desarrollo de la atención y el rendimiento académico en diversas áreas curriculares. La red de orientación mejora su funcionamiento a partir de los 6 años en tanto que la red ejecutiva tiene una importante estabilidad a partir de los 7 años y la red de alerta se optimiza a partir de los 10 años (Chang \& Burns, 2005; Rueda et al., 2004).

El desarrollo de la atención en el niño es un proceso gradual y evolutivo, que depende de una serie de factores. Con el paso del tiempo la atención se hace más organizada, flexible e independiente del medio (Colombo, 2001; Gómez Pérez \& Ostrosky Solís, 2006; Rueda, Posner \& Rothbart, 2005). De esta manera, la atención ofrece un indicador que permite predecir el nivel cognitivo en la infancia. Se ha comprobado tanto en niños como en adultos, que a mayor capacidad atencional hay una mejor ejecución en las tareas cognitivas (Chang \& Burns, 2005; Matute, Sanz, Gumá, Rosselli \& Ardila, 2009; Rosse1li \& Ardila, 2003). Asimismo, se ha demostrado que variables ambientales, culturales y educacionales tales como el nivel socioeconómico y el funcionamiento familiar y escolar modulan el desempeño en tareas que demandan atención y otros procesos de control cognitivo, ya que inciden en el bienestar del niño dado que determinan diferencias en el acceso a los recursos materiales y sociales (Ardila, Rosselli, Matute \& Guajardo, 2005; Bradley \& Corwyn, 2002; Lipina, Martelli, Vuelta \& Colombo, 2005; Lipina, Martelli, Vuelta, Injoque-Ricle \& Colombo, 2004; Matute et al., 2009).

La evaluación de la atención no es tarea sencilla debido a que acompaña a otras actividades cognitivas y puede fluctuar en un mismo sujeto dependiendo del momento, las circunstancias, el tipo de actividad, los intereses y la motivación, entre otros aspectos (Benedet, 2002). Así, las principales habili- 
dades perceptivo-atencionales empleadas en la evaluación de tareas atencionales son: (1) percepción de diferencias, (2) discriminación visual, (3) integración visual y (4) rapidez perceptiva. Estas tareas pueden ser caracterizadas como sencillas, sin embargo incluyen una gran cantidad de ensayos, lo que exige un período de tiempo relativamente amplio para ser realizadas. De esta manera se convierten en tareas de ejecución continua, en las que el sujeto ha de localizar los estímulos significativos entre un conjunto más amplio de estímulos que actuarían como distractores (Ison \& Carrada, 2011; Monteoliva, Ison \& Pattini, 2014). Dentro de los principios de percepción de diferencias, algunas de las pruebas más empleadas son: Test Formas Idénticas o FI (Thurstone, 1944), Test d-2 (Brickenkamp, 1981) y Test de Percepción de Semejanzas y Diferencias (TPSD) o CARAS (Thurstone \& Yela, 1985; 2012), entre otros.

La particularidad que presenta este último (Test de CARAS) es que ha sido ampliamente utilizado y estudiado en diversas investigaciones (Crespo-Eguílaz, Narbona, Peralta \& Repáraz, 2006; Ison \& Anta, 2006; Ison \& Carrada, 2011). En una de ellas, realizada previamente por los autores (Monteoliva, Ison \& Pattini, 2014), se proponen nuevos indicadores para la evaluación atencional a través del Test CARAS denominados: eficiencia atencional (FA) y rendimiento atencional (RA). Las expresiones matematicas derivadas de estos indicadores surgieron de la revisión cronológica de los avances producidos en este y otros instrumentos de evaluación (Ison \& Carrada, 2011) y de la necesidad de conocer con mayor precisión las capacidades de los sujetos evaluados. En este contexto se introduce también el término desempeño atencional, como aquel concepto integrador de los indicadores de eficacia atencional (EA) (Ison \& Carrada, 2008), la eficiencia atencional (FA) y el rendimiento atencional (RA) de un sujeto en tareas de búsqueda visual. A partir de ese estudio, se propuso una nueva modalidad de análisis contemplando diferentes aspectos intervinientes en tareas de búsqueda visual, utilizadas para evaluar el desempeño atencional. A continuación, se definen los conceptos mencionados para su mejor entendimiento.

Eficacia Atencional (EA): Como consecuencia de una serie de investigaciones y nuevos estudios de la atención desde diversos modelos teóricos, se formula un nuevo concepto denominado eficacia atencional (EA) (Ison \& Carrada, 2008, 2011) (1). A partir de este concepto, se evalúa la eficacia de un sujeto en tareas que implican la utilización conjunta de habilidades de sostenimiento y selectividad atencional. Es decir, permite evaluar la exactitud con la que un niño discrimina estímulos dentro de un conjunto de estímulos semejantes. Cabe aclarar que en la terminología empleada, los errores de omisión $\left(E_{\mathrm{O}}\right)$ corresponden a las figuras iguales al modelo no señaladas, mientras que los errores de comisión $\left(E_{\mathrm{c}}\right)$ son las figuras señaladas que difieren del modelo -mal señaladas-.

$$
E A=\frac{A}{I}=\frac{A}{A+E_{C}+E_{O}}
$$

donde $(E A)$ es la eficacia atencional, $(A)$ es el número de aciertos, $(I)$ es el número de intentos y $(E)$ los errores, tanto de omisión $\left(E_{\mathrm{O}}\right)$ como de comisión $\left(E_{\mathrm{c}}\right)$.

Eficiencia Atencional (FA): Este concepto está definido como la capacidad cognitiva de regular y optimizar el mecanismo atencional para seleccionar y mantener la atención durante un período prolongado de tiempo, utilizando los mínimos recursos de tiempo disponibles (Monteoliva, Ison \& Pattini, 2014; Monteoliva, Santillán \& Pattini, 2013 ) (2). Es decir, este nuevo concepto representa los aciertos en relación al tiempo requerido para ello:

$$
F A=\frac{A}{T^{0.2}}
$$

donde $(F A)$ es la eficiencia atencional, $(A)$ es el número de aciertos $\mathrm{y}(T)$ el tiempo total requerido para realizar la tarea.

La importancia de este indicador radica en incorporar y cuantificar el factor tiempo en períodos no limitados y prolongados de eje- 
cución (posterior a los 3 ó 6 minutos hasta finalizar la tarea). Con la incorporación del factor tiempo al concepto de efíciencia atencional $(F A)$, se complementa la precisión y producción de los sujetos -eficacia atencional $(E A)$ - con la capacidad de producir -eficiencia atencional $(F A)$-. Sin embargo, la $F A$ por sí sola no es totalmente representativa sino es acompañada por la $E A$. A su vez, sin la $F A$, el factor tiempo, en los períodos posteriores a tres hasta finalizar la tarea, no es incorporado al análisis. Por lo tanto, se propone generar un nuevo indicador que entrelace ambos conceptos, denominado rendimiento atencional $(R A)$.

Rendimiento Atencional (RA): Este nuevo indicador permite obtener un diagnóstico más preciso, ya que incorpora a los valores individuales de $E A$ y $F A$, en un valor unificado de la relación entre ambos y se lo define como la resultante del producto entre el nivel de eficacia y eficiencia atencional obtenido por un individuo en tareas de selección y sostenimiento atencional (Monteoliva, Ison \& Pattini, 2014) (3).

$$
R A=\frac{E A}{T^{0.2}}
$$

donde $(R A)$ es el rendimiento atencional, $(T)$ el tiempo requerido para realizar la tarea y $(E A)$ es la eficacia atencional del sujeto en la tarea.

Desde la Neuropsicología del Desarrollo el trabajo que se informa pretende brindar un aporte al campo de la medición en Psicología a partir de la elaboración de normas para el Test de Percepción de Semejanzas y Diferencias o CARAS (Thurstone \& Yela, 1985; 2012) en los diferentes indicadores de desempeño atencional en una muestra de 4.462 alumnos de 7 a 12 años, de establecimientos escolares públicos de nivel primario del ámbito urbano y urbano-marginal del Gran Mendoza (República Argentina). Este estudio constituye un aporte al conocimiento en el área de medición psicológica, brindando un instrumento validado y estandarizado en los contextos de aplicación.
Las tablas percentilares elaboradas muestran los rangos mínimos y máximos (25-75) esperables para cada edad, atendiendo a la zona de emplazamiento de las escuelas: urbanas y urbano-marginadas.

\section{MÉTOdo}

Se realizó una investigación de tipo descriptiva (Babbie, 2000) en la cual se empleó un diseño no experimental (Kerlinger \& Lee, 2002) y seccional-transversal (Sierra Bravo, 2003).

\section{PARTICIPANTES}

Participaron 4.462 escolares de 7 a 12 años (edad promedio $=9.53$ años; $D E=1.706)$ de segundo a séptimo grado, que concurrían a 17 establecimientos educativos de nivel primario pertenecientes a ámbitos urbanos y urbanomarginales del Gran Mendoza. La muestra de estandarización se obtuvo por muestreo aleatorio, estratificado con procedimientos de distribución proporcional (Aiken, 2003; Kerlinger \& Lee, 2002). Los criterios de inclusión para la participación de los niños fueron los siguientes: (1) contar con la autorización de los padres para que los niños participaran del estudio, (2) contar con la aceptación de los escolares para participar en el estudio, (3) que los niños tuvieran un rendimiento intelectual esperable para su edad, (4) que no hubiera antecedentes de alteraciones neurológicas, mentales, trastornos visuales o auditivos y / o tratamiento farmacológico, (5) no realizar la administración en condiciones de fatiga física, tensión emocional, sueño, hambre o cualquier otra condición que pudiera afectar la comprensión de las instrucciones o de los contenidos y (6) durante la evaluación colectiva, los niños debían permanecer sentados, en posición cómoda y suficientemente alejados de sus compañeros/as para evitar que elijan las mismas respuestas que los demás, molestar o ser molestados por éstos.

La muestra estuvo conformada por 2.279 mujeres $(51.1 \%)$ y 2.183 varones $(48.9 \%)$. El 
$50.8 \%$ (2.265) eran alumnos de escuelas urbanas y el $49.2 \%$ (2.197) lo eran de escuelas urbano-marginales. Los estadísticos correspondientes a la variable edad se presentan en la Tabla 1. Como se observa, existe una distribución proporcional entre las variables de ambas muestras.

\section{INSTRUMENTO}

\section{DESCRIPCIÓN DE LA TÉCNICA ORIGINAL}

El Test de Percepción de Semejanzas y Diferencias, también denominado $C A R A S$, fue elaborado por Thurstone (1944) y posteriormente adaptado por Thurstone y Yela (1985). Es una prueba empleada para evaluar la atención y la aptitud para percibir, rápida y correctamente, semejanzas, diferencias y patrones estimulantes parcialmente ordenados (Thurstone \& Yela, 2012). Consta de 60 bloques-estímulos, cada uno de ellos está formado por tres dibujos esquemáticos de caras (con trazos elementales representando la boca, ojos, cejas y pelo), una de las cuales es distinta (ver Gráfíco 1). La tarea consiste en determinar cuál es la cara diferente y tacharla. El resultado es expresado por una puntuación directa del número total de aciertos producidos en un tiempo de 3 minutos. La prueba es aplicable a partir de los 6-7 años en adelante y en todos los niveles socio-económico-culturales.

E1 Test CARAS ha sido ampliamente utilizado y estudiado en diversas investigaciones (Crespo-Eguílaz et al., 2006; Ison \& Anta, 2006), generando nuevas reinterpretaciones de los resultados y conjuntamente con la aparición de nuevas propuestas metodológicas en los tiempos de ejecución de la prueba. Los indicadores empleados en este estudio son los que integran el concepto de desempeño atencional: eficacia atencional $(E A)$, eficiencia atencional $(F A)$ y rendimiento atencional $(R A)$ [ver expresiones (1), (2) y (3)]. A partir de ellos se ofrecen los datos normativos para escolares argentinos según la categoría de escuelas: urbanas y urbano-marginales.

\section{Procedimiento}

Para realizar el trabajo se solicitaron los permisos correspondientes a las autoridades de la Dirección General de Escuelas del Gobierno de Mendoza. En cada establecimiento se trabajó en jornadas completas (turnos mañana y tarde), informando a los alumnos que su participación era voluntaria y que la tarea no sería calificada como correcta o incorrecta. Se establecieron consideraciones para la administración colectiva del instrumento (grado completo): (a) buena iluminación sobre el plano horizontal de trabajo y (b) determinado momento del día para su aplicación (turno mañana de 9:00 a 12:00 hs. y turno tarde de 14:00 a 17:00 hs.). La importancia del criterio seleccionado radica en evitar momentos de fatiga, somnolencia y poca motivación derivados de períodos posteriores a la realización de tareas o actividades por un período prolongado.

\section{ANÁLISIS DE DATOS}

Inicialmente se evaluó la variable género con la Prueba $t$, para conocer diferencias estadísticamente significativas en el desempeño atencional $(E A, F A$ y $R A$ ) entre ambos géneros en ambas categorías de escuelas. Posteriormente, se generaron las tablas con los datos percentilares, de los diferentes indicadores de desempeño atencional esperable de un niño de 7 a 12 años para los indicadores de $E A, F A$ y $R A$ en los tiempos: ( $t 1)$ los primeros 180 [seg], $(t 2)$ de los 180 [seg] hasta finalizar la tarea y $(T)$ el tiempo total de la tarea. Para la construcción de las mismas, se tomaron como criterio de construcción del rango, los percentiles 25,50 y 75 . De esta manera, se generó un rango del desempeño atencional esperable por edad, para facilitar la labor del profesional en una primera interpretación general de los resultados obtenidos.

\section{Resultados}

En relación al primer objetivo, se analizó la variable género en las categorías de escuela 
(urbanas y urbano-marginales) por medio de una Prueba $t$ de Student; en ella no se observaron diferencias estadísticamente significativas entre niños y niñas (ver Tabla 2). El resultado de la confección de los rangos percentilares se informa en las Tablas 3, 4, 5, 6, 7 y 8.

\section{Discusión}

Se presentan las normas del Test de Percepción de Semejanzas y Diferencias o CARAS para niños de 7 a 12 años de edad, diferenciando los indicadores de desempeño atencional a fin de lograr mayor precisión al evaluar el complejo mecanismo atencional.

Cuando un individuo realiza una tarea de búsqueda visual que implica un cierto grado de monotonía, operan en forma conjunta el sostenimiento atencional y la capacidad para discriminar correctamente los estímulos (Carrada, 2011). Sostener la atención implica, por definición, mantener el foco atencional durante un determinado lapso de tiempo. Así, al evaluar la atención con una tarea de búsqueda visual, simultáneamente se ponen en juego la capacidad para discriminar estímulos, codificarlos e inhibir los posibles distractores, sosteniendo el nivel de ejecución durante el tiempo que la tarea lo requiera. La incorporación de la variable tiempo es sumamente relevante porque permite una mejor ponderación del desempeño atencional de un niño en esta tipología de tareas. Así se observa claramente al analizar las diferentes tablas, el desempeño atencional en el indicador de eficiencia atencional $(F A)$ y en rendimiento atencional $(R A)$ se incrementa conforme al aumento de la edad de los niños. Este aspecto no se observa tan claramente al analizar los resultados del indicador de eficacia atencional $(E A)$. Sin embargo, cabe destacar que no fue el objetivo de este estudio profundizar en estas diferencias y sus análisis, sino generar datos normativos de los indicadores por edades. Actualmente hay estudios en ejecución que profundizarán estos resultados.

El trabajo que se informa presenta la limitación de ser el estudio de una muestra regio- nal de alumnos. Estos resultados provienen de alumnos de establecimientos escolares públicos de nivel primario de los ámbitos urbano y urbano-marginados del Gran Mendoza, por lo cual la interpretación de los mismos debe ser contextualizada a estos ámbitos. Además debe reconocerse la importancia de evaluaciones complementarias para un adecuado diagnóstico del desempeño atencional.

Desde esta perspectiva se considera que brinda mayor precisión a la hora de detectar dificultades atencionales en un niño. El desarrollo de la atención se considera esencial para el funcionamiento de otros procesos socio-cognitivos y es un indicador que permite predecir el desempeño en tareas que demanden control cognitivo (Ison, Korzeniowski, Segretin \& Lipina, 2015). Posner y Rothbart (2014) sostienen que de todos los factores que influyen sobre el aprendizaje, la atención al material aprendido sería uno de los más importantes, al facilitar el almacenamiento y recuperación de los contenidos de memoria, permitiendo la actualización de dichos contenidos cuando la situación lo requiera.

A partir de estudios previos se pueden realizar algunas consideraciones: (a) la eficacia atencional $(E A)$ implica la utilización conjunta de habilidades de sostenimiento y selectividad y permite evaluar la exactitud con la cual un niño discrimina estímulos dentro de un conjunto de patrones semejantes en un tiempo fijo, por ejemplo tres minutos. (b) En tanto el concepto de eficiencia atencional (FA) está definido como la capacidad cognitiva de regular y optimizar el mecanismo atencional para seleccionar y mantener la atención (eficacia atencional) durante un período prolongado de tiempo, utilizando los mínimos recursos de tiempo disponibles (Monteoliva, Santillan \& Pattini, 2013). Aquí la variable tiempo juega un rol importante al permitir diferenciar aquellos sujetos que con igual nivel de eficacia atencional se diferencian unos de otros por la velocidad de procesamiento del estímulo (precisión). Es decir, niños que necesitan más tiempo (o menos) para lograr el mismo nivel de precisión. (c) Finalmente, el concepto que integra a los anteriores es el de 
rendimiento atencional $(R A)$, entendido como el producto entre el nivel de eficacia y eficiencia atencional obtenido por un individuo en tareas de selección y sostenimiento atencional (Monteoliva, Ison \& Pattini, 2014).

La capacidad atencional es clave para un adecuado funcionamiento cognitivo; por consiguiente su evaluación, mediante la utiliza- ción de instrumentos válidos, confiables y adaptados a nuestra población escolar, resulta relevante a los fines de precisar el diagnóstico y adecuar programas de intervención psicoeducativos que interrumpan el ciclo de fracaso y frustración y contribuyan a aumentar el sentimiento de autoeficacia en el escolar (Ison \& Anta, 2006).

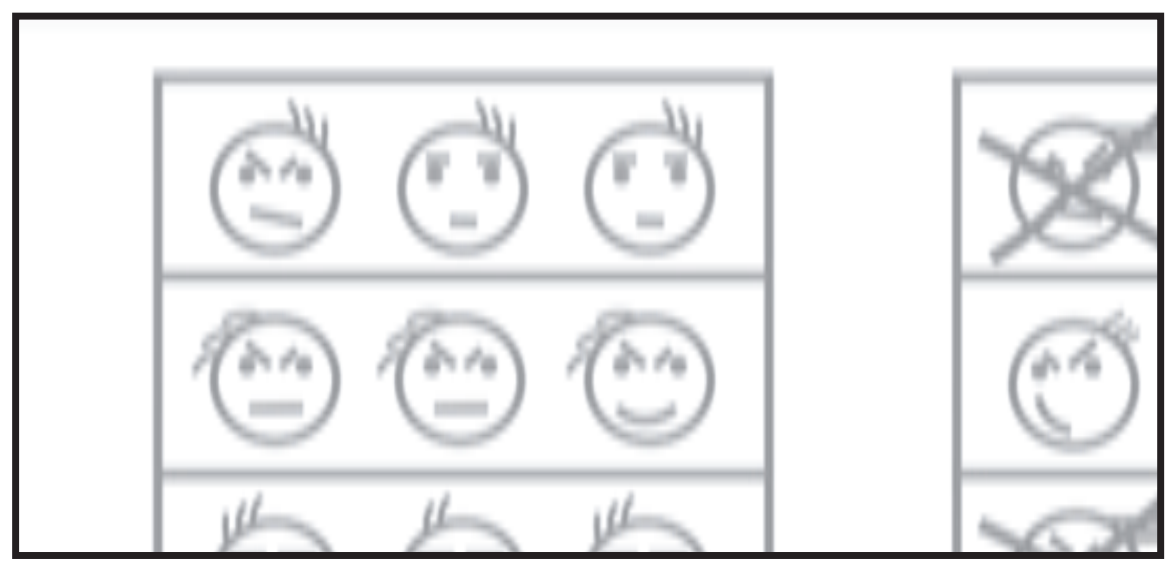


TABLA 1

ESTADÍSTICOS DESCRIPTIVOS DE LAS VARIABLES EDAD Y GÉNERO SEGÚN ZONA

\begin{tabular}{|c|c|c|c|c|c|c|c|c|}
\hline \multirow{3}{*}{$\begin{array}{r}\text { Edad } \\
\text { (años) }\end{array}$} & \multicolumn{4}{|c|}{ Urbana } & \multicolumn{4}{|c|}{ Urbano-marginal } \\
\hline & \multicolumn{2}{|c|}{ Mujer } & \multicolumn{2}{|c|}{ Hombre } & \multicolumn{2}{|c|}{ Mujer } & \multicolumn{2}{|c|}{ Hombre } \\
\hline & $f$ & $\%$ & $f$ & $\%$ & $f$ & $\%$ & $f$ & $\%$ \\
\hline 7 & 194 & 16 & 190 & 18 & 154 & 14 & 172 & 16 \\
\hline 8 & 189 & 16 & 200 & 19 & 183 & 17 & 190 & 17 \\
\hline 9 & 179 & 15 & 164 & 15 & 215 & 20 & 174 & 16 \\
\hline 10 & 218 & 18 & 155 & 14 & 174 & 16 & 189 & 17 \\
\hline 11 & 200 & 17 & 182 & 17 & 192 & 18 & 186 & 17 \\
\hline 12 & 210 & 18 & 184 & 17 & 172 & 16 & 196 & 18 \\
\hline$n$ & 1.190 & 100 & 1.075 & 100 & 1.090 & 100 & 1.107 & 100 \\
\hline$N$ & \multicolumn{4}{|c|}{2.265} & \multicolumn{4}{|c|}{2.197} \\
\hline
\end{tabular}

TABLA 2

Resultados de la prueba $t$ De Student, en la comparación de LA VARIAble GÉnERo SEgún zona

\begin{tabular}{|c|c|c|c|c|c|c|c|c|c|c|}
\hline \multirow{2}{*}{ Categoría } & \multicolumn{5}{|c|}{ Urbana } & \multicolumn{5}{|c|}{ Urbano-marginal } \\
\hline & $F$ & $t$ & $d f$ & $p$ & $M$ & $F$ & $t$ & $d f$ & $p$ & $M$ \\
\hline $\mathrm{EA}(\mathrm{t} 1)$ & .001 & 1.157 & 2263 & .247 & .005 & 1.434 & -1.856 & 2194 & .064 & .005 \\
\hline $\mathrm{EA}(\mathrm{t} 2)$ & .029 & -.215 & 2263 & .830 & -.001 & .581 & -.013 & 2195 & .990 & .007 \\
\hline $\mathrm{FA}(\mathrm{t} 2)$ & 2.064 & -2.186 & 2263 & .029 & -.007 & 1.001 & -.226 & 2195 & .821 & .003 \\
\hline $\mathrm{RA}(\mathrm{t} 2)$ & .195 & -.969 & 2263 & .333 & -.002 & .662 & .107 & 2195 & .915 & .003 \\
\hline $\mathrm{EA}(\mathrm{T})$ & .042 & .367 & 2263 & .713 & .002 & .270 & -.587 & 2195 & .557 & .006 \\
\hline $\mathrm{FA}(\mathrm{T})$ & .021 & -1.099 & 2263 & .272 & -.002 & .046 & -.127 & 2195 & .899 & .002 \\
\hline $\mathrm{RA}(\mathrm{T})$ & .377 & -.354 & 2263 & .724 & -.001 & 1.523 & -.473 & 2195 & .636 & .002 \\
\hline
\end{tabular}




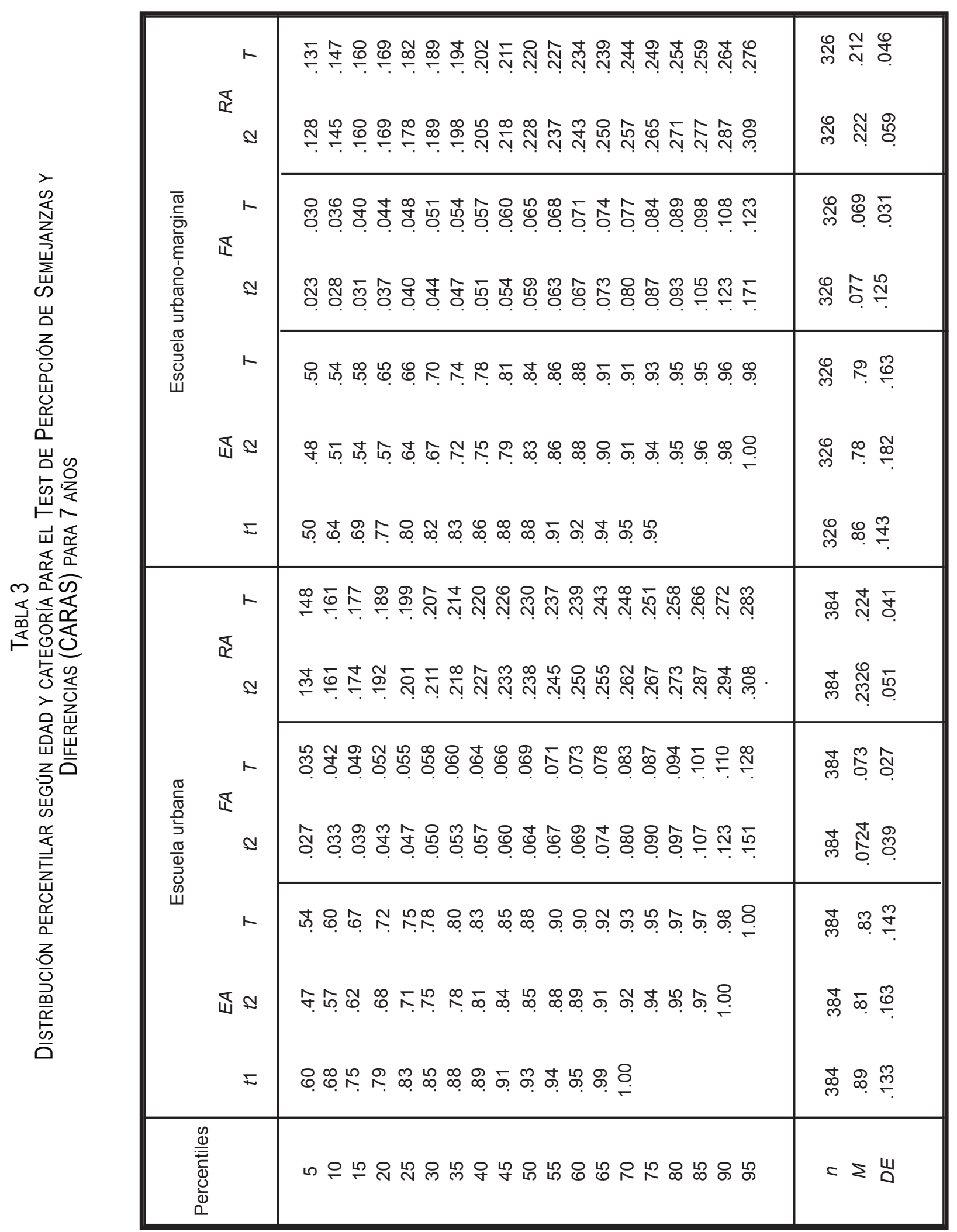




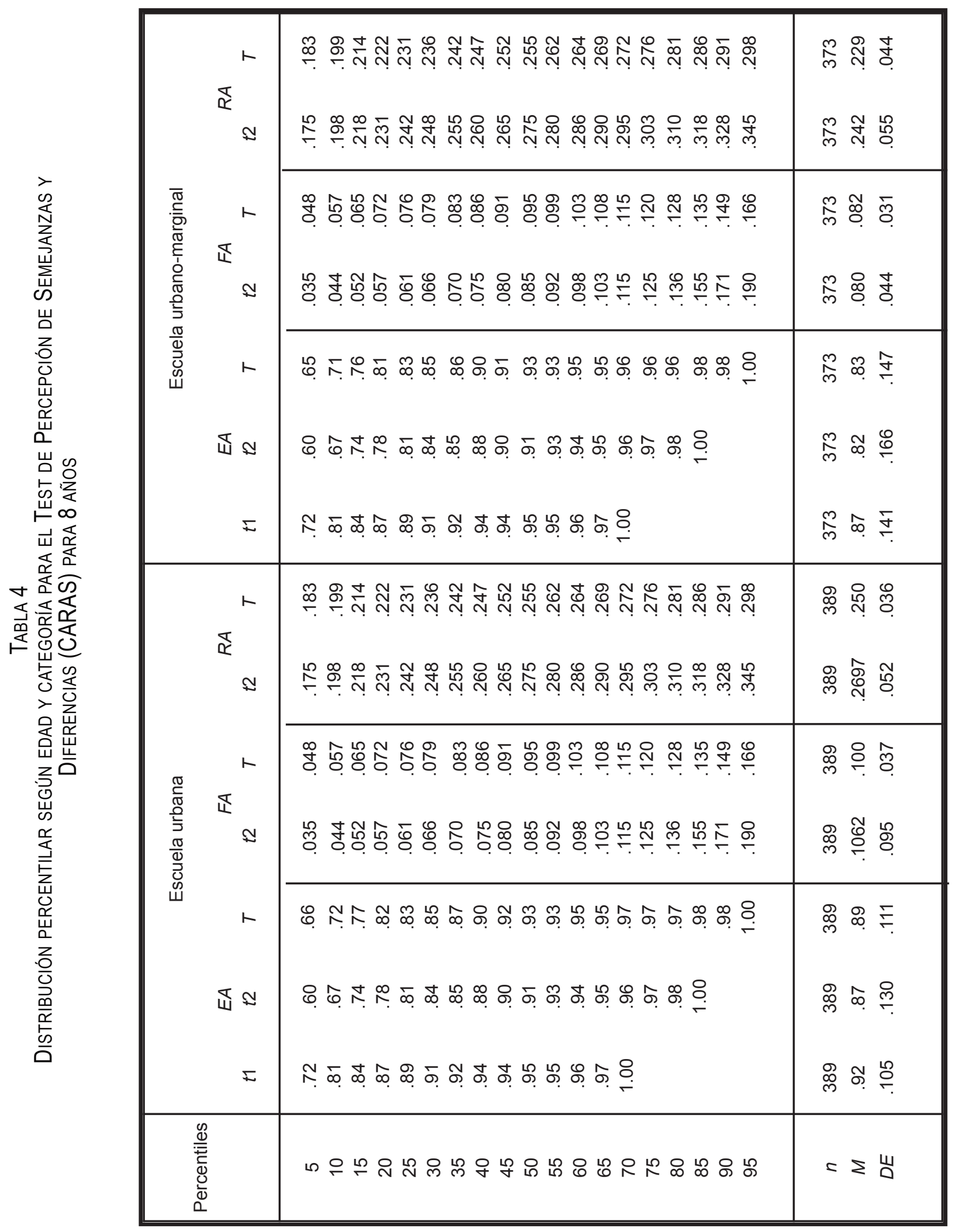




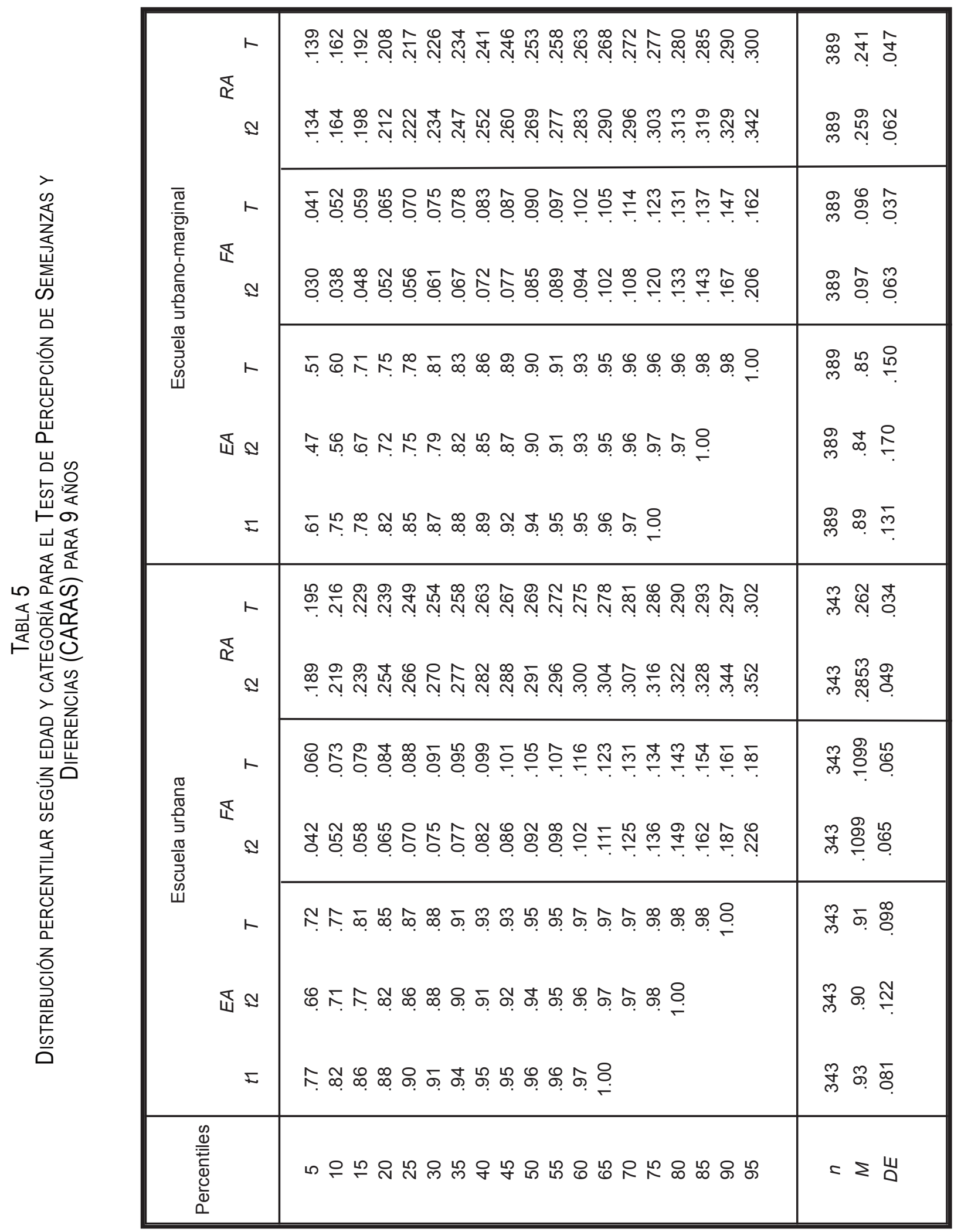




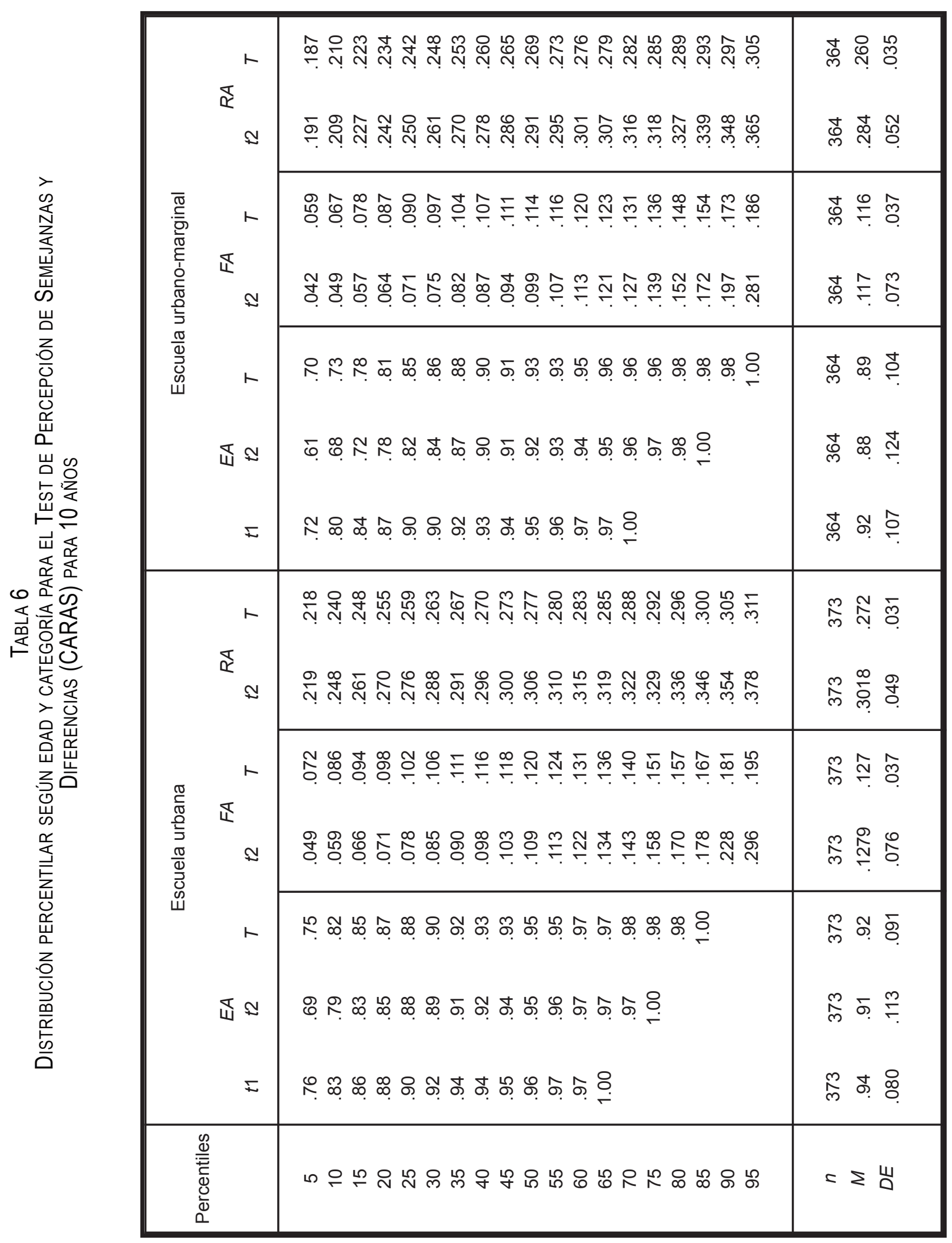




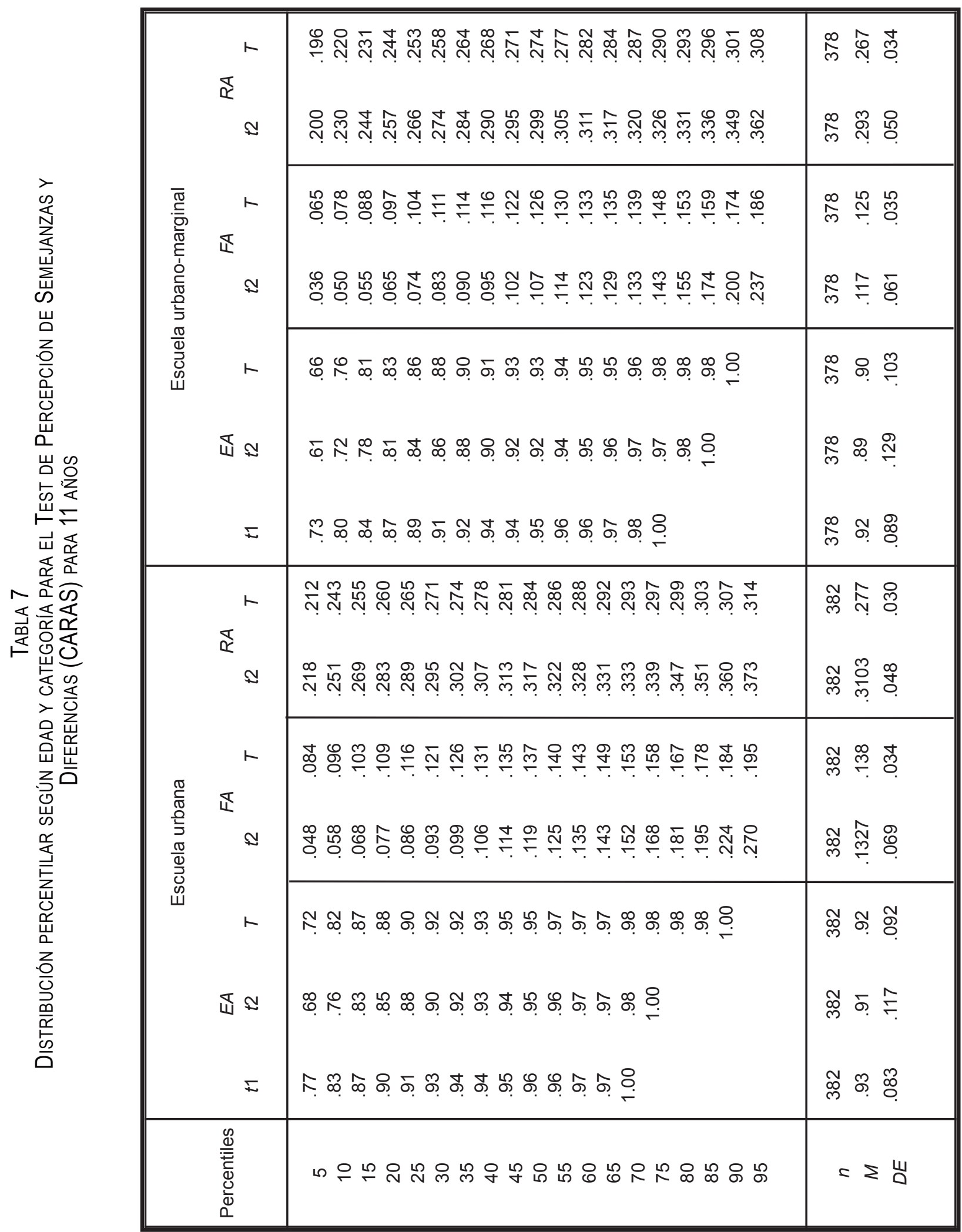




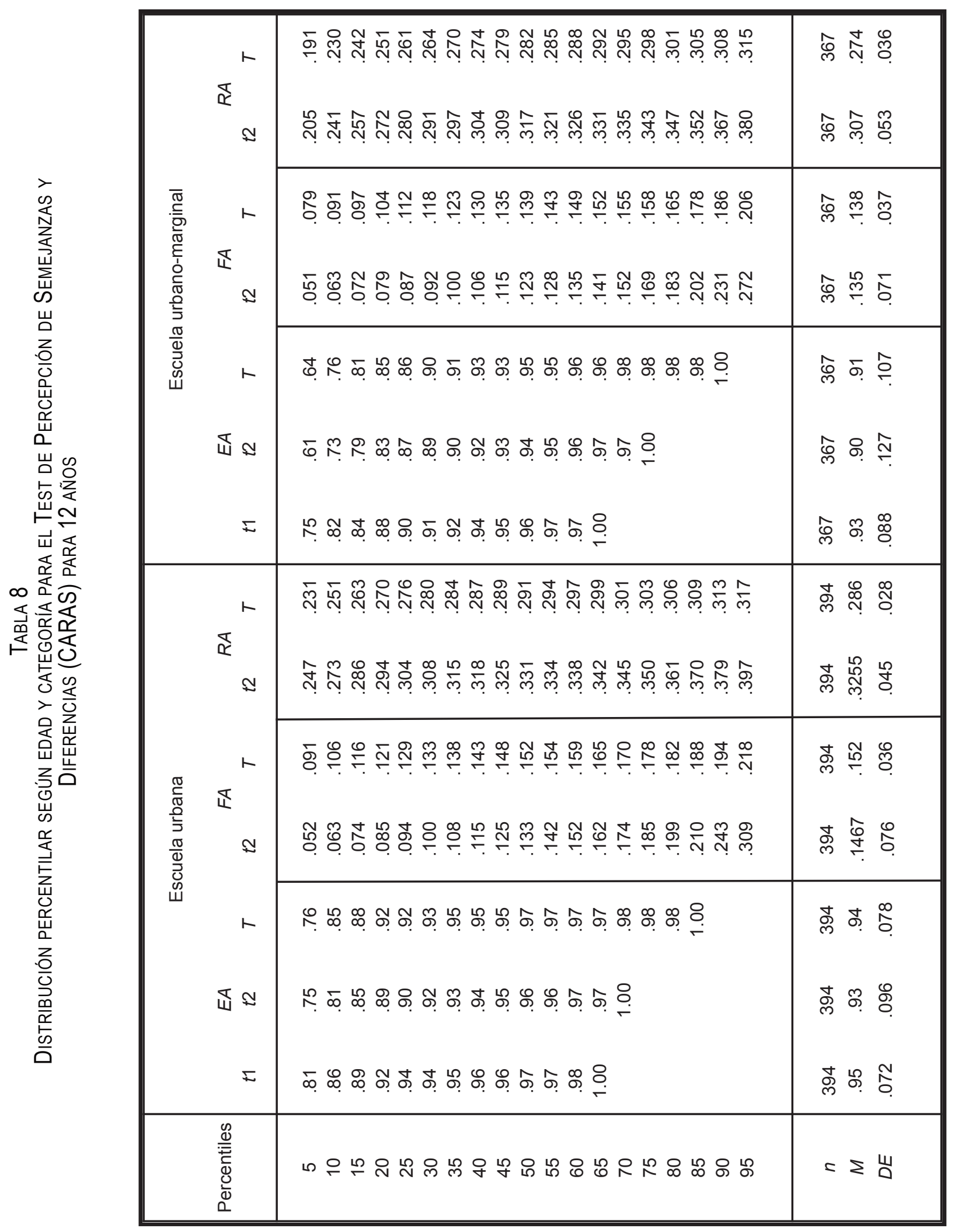




\section{REFERENCIAS BIBLIOGRÁFICAS}

Aiken, A. (2003). Test psicológicos y evaluación [Psychological test and evaluation]. México: Prentice Hall.

Álvarez, L., González-Castro, P., Núñez, J., Gonzáles Pienda, J., Álvarez, D. \& Bernardo, A. (2007a). Desarrollo de los procesos atencionales mediante actividades adaptadas [Development of attentional processes through adapted activities]. Papeles del Psicólogo, 28(3), 211217.

Álvarez, L., González-Castro, P., Núñez, J., González Pienda, J., Álvarez, D. \& Bernardo, A. (2007b). Programa de intervención multimodal para la mejora de los déficit de atención [Multimodal intervention program to improve attentional deficit]. Psicothema, 19, 590-595.

Álvarez, M. \& Trápaga, M. (2005). Principios de neurociencias para psicólogos [Neurosciences principles for Psychologists]. Buenos Aires: Paidós.

Ardila, A., Rosselli, M., Matute, E. \& Guajardo, S. (2005).The influence of the parents educational level on the development of executive functions. Developmental Neuropsychology, 28(1), 539-560.

Babbie, E. (2000). Fundamentos de la investigación social [Basics of social research]. México: Thomson.

Benedet, M.J. (2002). Neuropsicología cognitiva. Aplicaciones a la clínica y a la investigación. Fundamento teórico y metodológico de la neuropsicología cognitiva [Cognitive neuropsychology. Clinical applications and research. theoretical and methodological foundation of cognitive neuropsychology]. Madrid: INSERSO.

Bradley, R.H. \& Corwyn, R.F. (2002). Socioeconomic status and child development. Annual Review of Psychology, 53, 371-399.

Brickenkamp, R. (1981) Test d-2, Aufmerksamkeits-Belastungs Test [Attention Stress Test]. Göttingen, Germany: Hogrefe-Verlag.
Carrada, M.A. (2011). El mecanismo atencional en niños escolarizados: Baremación de instrumentos para su medición [The attentional mechanism in schoolchildren: Scale for measuring]. Facultad de Psicología, Universidad Nacional de San Luis, San Luis, Argentina.

Cassandra, B. \& Reynolds, C. (2005). A model of the development of frontal functioning: Findings from a meta-analysis. Applied Neuropsychology, 12(4), 190-201.

Chang, F. \& Burns, B. (2005). Attention in preeschoolers: Associations with effortful control and motivation. Child Development, 76(1), 247263.

Colombo J. (2001). The development of visual attention in infancy. Annual Review of Psycho$\log y, 52,337-367$.

Crespo-Eguílaz, N., Narbona, J., Peralta, F. \& Repáraz, R. (2006). Medida de atención sostenida y del control de la impulsividad en niños: Nueva modalidad de aplicación del Test de Percepción de Diferencias CARAS [Measure sustained attention and impulse control in children: A new method of applying the Differences Perception Test FACES]. Infancia y Aprendizaje, 29, 219-232.

Fuentes Melero, L.J. (2004). Inhibitory processing in the attentional networks. En M.I. Posner (Ed.), Cognitive neuroscience of attention (pp. 45-55). NY: Guilford Press.

Fuentes Melero, L.J. \& Campoy, G. (2008). The time course of alerting effectover orienting in the attentional networks test. Experimental Brain Research, 185, 667-672.

Fuentes Melero, L.J. \& García Sevilla, J. (2008). Manual de psicología de la atención: Una perspectiva neurocientifica [Manual of psychology of attention: A neuroscientific perspective]. Madrid: Síntesis.

Gómez Pérez, E. \& Ostrosky Solís, F. (2006). Attention and memory evaluation across the life span: Heterogeneous effects of age and education. Journal of Clinical and Experimental Neuropsychology, 28, 477-494. 
Ison, M.S., Korzeniowski, C., Segretin, M.S. \& Lipina, S.J. (2015). Evaluación de la eficacia atencional en niños argentinos sin y con extra-edad escolar [Assessment of attentional efficiency in Argentine schoolchildren with and without overage]. Revista Argentina de Ciencias del Comportamiento, 7(1), 38-52.

Ison, M.S. \& Anta, F.G. (2006). Estudio normativo del CARAS en niños mendocinos [Normative study of FACES in Mendoza's children]. Interdisciplinaria, 23(2), 203-231.

Ison, M.S. \& Carrada, M. (2011). Evaluación de la eficacia atencional: Estudio normativo preliminar en escolares argentinos [Assessment of attentional efficiency: Preliminary normative in students in Argentine]. RIPED, 29, 129-146.

Ison, M.S. \& Carrada, M.A. (Julio, 2008). Assessment of attentional efficiency: Preliminary normative study carried out with students in Mendoza [Resumen]. Journal of the International Neuropsychological Society, 14(2), 11. Recuperado el 21 de marzo de 2013 de https:/ www. the-ins.org/documents/Abstract_MY 08. pdf

Ison, M.S., Greco, C., Korzeniowski, C. \& Morelato, G.S. (2015). Selective attention: A comparative study on Agentine students from different socioeconomic contexts. Electronic Journal of Research in Educational Psychology, 13 (2), 343-368. http://dx.doi.org/10.142 04/ejrep. 36.14092

Kerlinger, F. \& Lee, H. (2002). Investigación del comportamiento [Behavioral research]. México: McGraw-Hill.

Korzeniowski, C. (2011). Desarrollo evolutivo del funcionamiento ejecutivo y su relación con el aprendizaje escolar [Evolutionary development of executive functioning and its relationship with school learning]. Revista de Psicologia, 7 (13), 7-26.

Lipina, S.J., Martelli, M.I., Vuelta, B., Injoque Ricle, I. \& Colombo, J.A. (2004). Pobreza y desempeño ejecutivo en alumnos preescolares de la ciudad de Buenos Aires (Argentina) [Poverty and educational performance in preschoolers in the city of Buenos Aires (Argentina)]. Interdisciplinaria, 21(2), 153-193.

Lipina, S.J., Martelli, M.I., Vuelta, B.L. \& Colombo, J.A. (2005). Performance on the A-not-Btask of Argentinean infants from Unsatisfied Basic Needs Homes. Interamerican Journal of Psychology, 39, 49-60.

Matute, E., Sanz, A., Gumá, E., Rosselli, M. \& Ardila, A. (2009). Influencia del nivel educativo de los padres, el tipo de escuela y el sexo en el desarrollo de la atención y la memoria [Influence of educational level of parents, school type and sex in the development of attention and memory]. Revista Latinoamericana de Psicología, 41(2), 257-276.

Monteoliva, J.M., Santillan, J. \& Pattini, A. (2013). Eficacia atencional en el contexto educativo: Evaluación de dos tests basados en tareas visuales [Attentional efficiency in the educational context: Evaluation of two tests based on visual tasks]. Actas del XXXIV Congreso Interamericano de Psicología (SIP). Sociedad Interamericana de Psicología (pp. 15-19). Universidade de Brasilia. Centro Universitarario de Brasilia. Brasilia, Brasil.

Monteoliva, J.M., Ison, M.S. \& Pattini, A.E. (2014). Evaluación del desempeño atencional en niños: Eficacia, eficiencia y rendimiento [Evaluation of attentional performance in children: Efficacy, efficiency and yield]. Interdisciplinaria, 31(2), 213-225.

Petersen, S.E. \& Posner, M.I. (2012). The attention system of the human brain: 20 years after. Annual Review Neuroscience, 35, 73-89. http:// dx.doi.org/10.1146/annurev-neuro-062111-150 525. Epub 2012 Apr 12.

Portellano, J. (2007). Neuropsicología infantil [Child neuropsychology]. España: Editorial Síntesis.

Posner, M.I. \& Rothbart, M.K. (2014). Attention to learning of school subjects. Trends in Neuroscience and Education, 3(1), 14-17. http://dx. doi.org/10.1016/j.tine.2014.02.003i 
Rosselli, M. \& Ardila, A. (2003). The impact of culture and education on nonverbal neuropsychological measures: A critical review. Brain and Cognition, 52, 326-33.

Rosselló i Mir, J. (1998). Psicología de la atención. Introducción al estudio del mecanismo atencional [Psychology of attention: Introduction to the study of the attention mechanism]. Madrid: Pirámide Ediciones.

Rueda, M.R., Fan, J., McCandliss, B.D., Halparin, J.D., Gruber, D.B., Pappert Lercari, L. \& Posner, M.I. (2004). Development of attentional networks in childhood. Neuropsychologia, 42, 1029-1040.

Rueda, M.R., Posner, M.I. \& Rothbart, M. (2005). The development of executive attention: Contributions to the emergence of self-regulation.
Developmental Neuropsychology, 28(2), 573594.

Sierra Bravo, R. (2003). Técnicas de investigación social. Teorías y ejercicios [Social research techniques. Theories and exercises] (14a. ed.). Madrid: Thomson.

Thurstone, L.L. (1944). A factorial study of perception. Chicago: The University of Chicago Press.

Thurstone, L.L. \& Yela, M. (1985). CARAS - Test de Percepción de diferencias [FACES - Perception of differences test]. Buenos Aires: TEA Ediciones.

Thurstone, L.L. \& Yela, M. (2012). CARAS-R Test de Percepción de Diferencias [FACES-R Perception of differences test]. Madrid: TEA Ediciones.
Instituto de Ciencias Humanas Sociales y
Ambientales (INCIHUSA)
Consejo Nacional de Investigaciones
Cientificas y Técnicas (CONICET)
Mendoza - República Argentina

Fecha de recepción: 28 de septiembre de 2015 Fecha de aceptación: 4 de julio de 2016 